\title{
Erratum to: Precise orbit determination and accuracy analysis of HY-2A satellite using DORIS Doppler data
}

\author{
Qiaoli Kong · Jinyun Guo • Cheinway Hwang • \\ Fan Gao - Shinfa Lin - Chunmei Zhao
}

Published online: 30 July 2014

(C) Akadémiai Kiadó 2014

\section{Erratum to: Acta Geod Geophys DOI 10.1007/s40328-014-0066-4}

The fifth author's name was incorrect in the original publication. The name is correct in this erratum.

The online version of the original article can be found under doi:10.1007/s40328-014-0066-4.

Q. Kong · J. Guo (区)

State Key Laboratory of Mining Disaster Prevention and Control Co-founded by Shandong Province and the Ministry of Science and Technology, Shandong University of Science and Technology,

Qingdao 266590, China

e-mail: jinyunguo1@126.com

Q. Kong · J. Guo

Key Laboratory of Surveying and Mapping Technology on Island and Reef, State Bureau of Surveying and Mapping, 579 Qianwangang Road, Qingdao, China

e-mail: kqlabc3334@hotmail.com

C. Hwang $\cdot$ S. Lin

Department of Civil Engineering, National Chiao Tung University, 1001 University Road, Hsinchu, Taiwan

e-mail: cheinway@mail.nctu.edu.tw

F. Gao

Institute of Geodesy and Geophysics, Chinese Academy of Sciences, 340 XuDong Road, Wuhan, China

C. Zhao

Chinese Academy of Surveying and Mapping, 28 Lianhuachixi Road, Beijing, China 